\section{Tendência da proporção de baixo peso ao nascer, no período de 1994-2004, por microrregião do Rio Grande do Sul, Brasil: uma análise multinível}

\author{
Trends in the proportion of low birth weight from \\ 1994 to 2004 in Rio Grande do Sul State, Brazil: \\ a multilevel analysis
}

\author{
1 Universidade Federal de \\ Santa Maria, Santa Maria, \\ Brasil. \\ 2 Universidade Federal do Rio \\ Grande do Sul, Porto Alegre, \\ Brasil. \\ Correspondência \\ A. B. Moraes \\ Universidade Federal de \\ Santa Maria. \\ Rua Conde de Porto Alegre \\ 1080, apto. 301, Santa Maria, \\ RS 97015-110, Brasil. \\ anaelena.bm@terra.com.br
}

\begin{abstract}
The aim of this longitudinal ecological study was to analyze the trend in the proportion of low birth weight in Rio Grande do Sul State, Brazil, from 1994 to 2004 by panel data analysis and multilevel linear regression (two levels: by microregion and time in years) to estimate risk factors associated with low birth weight. The proportion of low birth weight increased by $1.2 \%$ per year, and the multilevel model showed that the proportions differed between the micro-regions and increased over time, with the increase in the percentage of premature newborns, with the increase in the infant mortality rate, and with the increase in the cesarean rate. Among the microregions, the proportions of low birth weight varied positively with the urbanization rate and expenditures in the Unified National Health System and negatively with rate of participation in the workforce. According to the multilevel model, most of the variation in proportions of low birth weight was due to the effects of the micro-region of residence of the newborn's mother.
\end{abstract}

Information Systems; Low Birth Weight; Multilevel Analysis

\author{
Anaelena Bragança de Moraes 1 \\ Roselaine Ruviaro Zanini 1 \\ Elsa Regina Justo Giugliani 2 \\ João Riboldi 2
}

\section{Introdução}

O peso ao nascer assim como a mortalidade infantil constituem excelentes indicadores socioeconômicos. Existem dois principais mecanismos que levam ao baixo peso (peso $<2.500 \mathrm{~g}$ ): a prematuridade (idade gestacional $<37$ semanas) e o crescimento intrauterino restrito, também conhecido como desnutrição fetal, que ocorre quando a criança nasce com peso abaixo do valor-limite para a sua idade gestacional 1 .

O ponto de corte para o baixo peso ao nascer adotado para comparações internacionais é baseado em observações epidemiológicas, as quais indicam que crianças pesando menos de $2.500 \mathrm{~g}$ têm, aproximadamente, 20 vezes mais chance de morrer do que bebês que nascem com mais peso ${ }^{1,2}$. O baixo peso ao nascer, mais comum em países em desenvolvimento, contribui para uma variedade de desfechos desfavoráveis na saúde dos recém-nascidos ${ }^{3}$.

A redução de no mínimo um terço na incidência de baixo peso ao nascer entre os anos de 2000 e 2010 é um dos principais objetivos da World Fit for Children, na Declaração e Plano de Ação adotados na Sessão Especial sobre Crianças, na Assembléia Geral das Nações Unidas, em 2002. A redução da incidência de baixo peso ao nascer contribui para que seja atingido o Objetivo de Desenvolvimento do Milênio para a redução da mortalidade infantil (http://www.unicef.pt/ pagina_relatorio.php, acessado em 25/Jul/2006). 
As proporções de baixo peso ao nascer no Brasil, entre 1994 e 2002, não apresentaram grande variação. Entre 1996 e 2000, houve uma redução de $1,8 \%$, e entre 2000 e 2006 houve um acréscimo de $6,2 \%$, sendo $8,1 \%$ em 2006. A Região Sudeste foi a que apresentou maior proporção nesse período, com percentual de $9,1 \%$ para 2006 , seguida da Região Sul com 8,5\% para o mesmo ano. No Rio Grande do Sul, os valores foram 8,7\%; 9\%; 9,3\%; $9,5 \%, 9,3 \%, 9,1 \%$ e $9,1 \%$ para os anos de 2000 2006, respectivamente, ficando acima do valor médio no Brasil, que é $8 \% 4$ (Informações de saúde: estatísticas vitais. http://portal.saude.gov.br/ portal/svs/visualizar_texto.cfm?idtxt $=24455$ n, acessado em 30/Mar/2010; http://www.datasus. gov.br, acessado em 29/Jul/2006).

A avaliação do comportamento das proporções de baixo peso ao nascer nas localidades e no tempo e das associações com indicadores socioeconômicos, de saúde, de renda etc. dessas localidades, pode levar a uma melhor compreensão das proporções de baixo peso ao nascer como indicador coletivo de saúde.

Recentes avanços na área de estatística, particularmente os denominados modelos multiníveis de efeitos aleatórios, trazem perspectivas inovadoras para a análise de dados, com a inclusão das análises hierárquicas complementando e superando as análises em nível individual. Grande parte da pesquisa atual em epidemiologia é baseada no individualismo metodológico 5 .

O objetivo deste estudo foi identificar preditores para a proporção de baixo peso ao nascer de crianças nascidas no Estado do Rio Grande do Sul, no período de 1994-2004, utilizando um modelo de regressão linear multinível. Como variáveis preditoras, foram utilizados os indicadores das microrregiões nos anos considerados, sendo os anos, o nível individual, e as microrregiões, o nível contextual. Os resultados foram comparados ao do modelo de dados de painel.

\section{Método}

Este é um estudo ecológico longitudinal em que foram avaliadas as proporções de baixo peso ao nascer dos recém-nascidos oriundos das $35 \mathrm{mi}$ crorregiões do Rio Grande do Sul, nos anos de 1994-2004.

O Estado do Rio Grande do Sul tem Porto Alegre como capital e está dividido, geograficamente, em 7 mesorregiões, 35 microrregiões e 496 municípios (Instituto Brasileiro de Geografia e Estatística. http://www.ibge.gov.br, acessado em 10/Mai/2005). Uma microrregião é, de acordo com a Constituição Federal de 1988, um agrupamento de municípios limítrofes.
Os indicadores considerados como variáveis preditoras da proporção de baixo peso ao nascer, do nível individual e contextual, foram selecionados de acordo com a disponibilidade das informações para as microrregiões no período considerado e com base na revisão da literatura. Os indicadores com informações disponíveis para os 11 anos do estudo foram considerados no nível individual. Os indicadores utilizados no nível da microrregião foram aqueles que só possuíam informações para o ano do Censo Demográfico 2000, e/ou de um ano mais recente (2003 e/ou 2004). Alguns indicadores foram utilizados nos dois níveis.

A variável desfecho foi a proporção de baixo peso ao nascer nas 35 microrregiões nos 11 anos, totalizando 385 valores percentuais, disponíveis no SINASC (Sistema de Informações sobre Nascidos Vivos - Departamento de Informática do SUS. http://www.datasus.gov.br).

A proporção de baixo peso ao nascer, por definição, é uma variável derivada, que sumariza características de indivíduos em grupos, podendo ser tratada no nível individual ou contextual 6 . Neste estudo, ela é de nível individual.

O nível individual compreende os 11 anos do período do estudo. As covariáveis de interesse, disponíveis no SINASC para os anos de 19942004, para todas as microrregiões foram: nascidos vivos pré-termo (percentual de nascidos vivos com idade gestacional menor que 37 semanas), coeficiente de mortalidade infantil (número de óbitos de menores de um ano dividido pelo número de nascidos vivos num determinado espaço geográfico e ano considerado, por mil nascidos vivos), taxa de sete ou mais consultas prénatais (percentual de nascidos vivos de mães que realizaram sete ou mais consultas pré-natais) e taxa de cesáreas (percentual de partos cesáreos em relação ao total de partos).

O nível contextual compreende as microrregiões. Os indicadores foram obtidos no DATASUS (http://www.datasus.gov.br, acessado em Dez/ 2005), na Fundação de Economia e Estatística (FEEdados; http://www.fee.rs.gov.br/feedados, acessado em Dez/2005), vinculada à Secretaria da Coordenação e Planejamento do Rio Grande do Sul e Instituto de Pesquisa Econômica Aplicada (IPEA; http://www.ipeadata.gov.br, acessado em Dez/2005), vinculado ao Ministério de Planejamento, Orçamento e Gestão.

Os indicadores utilizados como covariáveis das microrregiões foram: taxa de urbanização (percentual da população da área urbana em relação à população total); taxa de analfabetismo (percentual da população de pessoas com 15 anos ou mais de idade, incapazes de ler ou escrever um bilhete simples); média de anos de 
estudo (razão entre o somatório do número de anos de estudo completados pelas pessoas com 25 anos ou mais de idade e o número de pessoas nessa faixa etária); PIB per capita (Produto Interno Bruto a preço de mercado dividido pela população); taxa de pobreza (percentual da população com renda domiciliar per capita inferior a meio salário mínimo); densidade por domicílio (percentual de pessoas que vivem em domicílios com densidade superior a 2, dada pela razão entre o total de moradores do domicílio e o número total de cômodos, excluídos o(s) banheiro(s) e mais um cômodo, destinado à cozinha); taxa de homicídios (razão entre o número de homicídios e o número de pessoas da população por 10 mil habitantes); taxa de internações por agressão (razão entre o número de internações hospitalares por agressão e o número de pessoas da população por $100 \mathrm{mil}$ habitantes); taxa de participação (percentual da população economicamente ativa entre a população em idade produtiva); coeficiente de mortalidade infantil; taxa de cobertura da Estratégia Saúde da Família (ESF) (percentual de pessoas cadastradas em relação à população); abastecimento de água (percentual de domicílios com água disponibilizada por rede geral); saneamento (percentual de domicílios com instalações sanitárias ligadas à rede geral); taxa de cesáreas (percentual de partos cesáreos em relação ao total de partos); taxa de sete ou mais consultas pré-natais (percentual de nascidos vivos de mães que realizaram sete ou mais consultas pré-natais); cobertura de saúde suplementar (percentual de beneficiários de assistência médica privada e por convênios, por local de residência); cobertura de médicos (proporção de médicos por mil habitantes, incluindo os médicos residentes); gasto médio com atenção básica (valores de transferências referentes à atenção básica por habitante); IDESE (índice de desenvolvimento socioeconômico que abrange quatro blocos temáticos: educação, renda, saneamento e domicílios e saúde).

O coeficiente de mortalidade infantil foi utilizado, neste estudo, como um indicador das condições de saúde da microrregião, por ser, ao longo do tempo, considerado não só como um bom indicador do nível de saúde, mas também como indicador das condições de vida de uma população 7 .

Para facilitar a interpretação dos coeficientes estimados pelos modelos de medidas repetidas e multinível, as covariáveis foram consideradas na forma contínua, centradas na média ${ }^{8}$.

Para estimar a taxa de evolução da proporção de baixo peso ao nascer das microrregiões, foi utilizado o modelo exponencial, ajustado aos va- lores anuais da proporção de baixo peso ao nascer nos 11 anos.

A análise de dados de painel via modelos mistos possibilitou a consideração de diferentes estruturas de covariância, selecionando-se a estrutura mais adequada para a variação de medidas dentro de indivíduos. As microrregiões foram consideradas os indivíduos, e os anos, o efeito de repetição. A variável resposta foi a proporção de baixo peso ao nascer. A melhor estrutura de covariância usada para estimar os coeficientes do modelo foi a que apresentou os menores valores para os critérios AIC (Critério de Informação de Akaike) e BIC (Critério Bayesiano de Schwarz) ${ }^{9}$. O melhor modelo foi selecionado pelo teste da razão de verossimilhança (deviance).

Para avaliar possíveis problemas de multicolinearidade nos modelos, foi realizada a análise de correlação entre as variáveis preditoras.

Como modelo multinível, foi empregado um modelo linear hierárquico com dois níveis para identificar preditores da proporção de baixo peso ao nascer, levando-se em conta a variação entre os anos (nível 1) e a variação entre as microrregiões (nível 2).

Inicialmente, foi ajustado aos dados um modelo de componentes de variância (intercepto aleatório), onde $y_{i j}$ é a variável resposta (contínua), $X_{1}, X_{2}, \ldots, X_{p}$ são as variáveis aleatórias no nível individual (nível 1), e $Z_{1}, Z_{2}, \ldots, Z_{q}$ são as variáveis no nível do grupo ou contextual (nível 2) 10:

$\mathrm{y}_{\mathrm{ij}}=\beta_{0}+\beta_{10} \mathrm{x}_{\mathrm{lij}}+\ldots+\beta_{\mathrm{p} 0} \mathrm{x}_{\mathrm{pij}}+\beta_{01} \mathrm{z}_{1 \mathrm{j}}+\ldots+\beta_{\mathrm{oq}} \mathrm{z}_{\mathrm{qj}}+\mathrm{u}_{0 \mathrm{j}}+\mathrm{e}_{0 \mathrm{ij}}(1)$

Os parâmetros fixos $\beta_{\mathrm{h} 0}$ e $\beta_{0 \mathrm{~h}}$ dos níveis $1 \mathrm{e}$ 2 , respectivamente, têm a mesma interpretação dos coeficientes no modelo de regressão múltipla. A parte aleatória do modelo é formada pelos termos de erros $\mathrm{u}_{\mathrm{oj}}$ (nível 2) e $\mathrm{e}_{\mathrm{ij}}$ (nível 1), mutuamente independentes, com média zero e variância $\sigma_{\mathrm{u} 0}^{2}$ e $\sigma_{\mathrm{e} 0}^{2}$, respectivamente 10 .

O modelo de coeficientes aleatórios, em que o intercepto e a inclinação são aleatórios, é representado aqui considerando a variável resposta $\mathrm{Y}$ e apenas uma variável explanatória $X$, medida no nível 1 para facilitar a demonstração:

$$
y_{i j}=\beta_{0 j}+\beta_{1 j} x_{i j}+e_{0 i j}
$$

em que: $\beta_{0 \mathrm{j}}$ é o intercepto do j-ésimo grupo do nível $2 ; \beta_{1 \mathrm{j}}$ é a inclinação associada à variável explanatória $\mathrm{x}_{\mathrm{ij}}$ da i-ésima unidade do nível 1 para o j-ésimo grupo do nível 2.

Para o nível 2, podem-se definir j modelos similares, e para cada um deles há diferentes interceptos $\beta_{0 \mathrm{j}}$ e inclinações $\beta_{1 \mathrm{j}}$.

Estes coeficientes de regressão são considerados como variáveis respostas, dadas por: 
$\beta_{0 \mathrm{j}}=\beta_{0}+\mathrm{u}_{0 \mathrm{j} ;} \beta_{1 \mathrm{j}}=\beta_{1}+\mathrm{u}_{1 \mathrm{j}}$, em que $\beta_{0}$ é a média dos interceptos no nível 2; $\beta_{1}$ é a média das inclinações no nível $2 ; \mathrm{u}_{0 \mathrm{j}}$ e $\mathrm{u}_{1 \mathrm{j}}$ são variáveis aleatórias com média 0 , variância $\sigma_{\mathrm{u} 0}^{2}, \sigma_{\mathrm{u} 1}^{2}$ e covariância $\sigma_{\mathrm{u} 01}$ em que $\sigma_{\mathrm{u} 0}^{2}$ representa a variabilidade do intercepto, e $\sigma_{\mathrm{u} 1}^{2}$ representa a variabilidade das inclinações entre os grupos 10 .

A equação (2) pode ser escrita na forma:

$$
\begin{gathered}
\mathrm{y}_{\mathrm{ij}}=\beta_{\mathrm{o}}+\beta_{1} \mathrm{x}_{\mathrm{ij}}+\left(\mathrm{u}_{0 \mathrm{j}+} \mathrm{u}_{1 \mathrm{j}} \mathrm{x}_{\mathrm{ij}+} \mathrm{e}_{\mathrm{ij}}\right) \\
\operatorname{var}\left(\mathrm{e}_{0 \mathrm{ij}}\right)=\sigma_{\mathrm{e} 0}^{2}
\end{gathered}
$$

A variável resposta $y_{\mathrm{ij}}$ é expressa como a soma de uma parte fixa e uma parte aleatória, dentro dos parênteses 10 .

Os modelos multiníveis foram estimados por Mínimos Quadrados Iterativos Generalizados (IGLS) 11.

Com o objetivo de medir a correlação intragrupo $(\rho)$, que expressa a proporção da variância total devido ao nível 2, foi avaliado o modelo multinível nulo com o intercepto aleatório. A correlação intragrupo, em um modelo de dois níveis, também é a correlação entre duas unidades do nível 1 dentro de um mesmo grupo do nível 2 e é calculada por:

$$
\rho=\sigma_{\mathrm{u} 0}^{2} /\left(\sigma_{\mathrm{u} 0}^{2}+\sigma_{\mathrm{e} 0}^{2}\right)
$$

em que $\sigma^{2}{ }_{\mathrm{u} 0}$ é a variância entre as unidades do nível 2, e $\sigma^{2}$ e0 é a variância entre as unidades do nível 111.

Posteriormente, o modelo foi avaliado só com a variável anos, centrada no ano de 1994, com o objetivo de avaliar o comportamento da proporção de baixo peso ao nascer no tempo.

Foi testado um termo quadrático para a variável anos, pois, segundo Leyland \& McLeod 12, o pressuposto de uma tendência linear poderia ser simplista.

Para a seleção das variáveis do nível 2, todas as variáveis contextuais foram avaliadas individualmente no modelo com a variável anos, para a verificação da significância estatística ( $\mathrm{p}<0,25)$. Posteriormente, foram incluídas as variáveis significativas do nível 1 e do nível 2, para a avaliação da significância conjunta e da deviance 13 .

A significância estatística dos coeficientes da parte fixa e da parte aleatória do modelo multinível foi avaliada pelo teste de Wald, a 5\% de significância.

A variância do nível 1 e do nível 2 foi modelada em função das covariáveis preditoras, resultando em um modelo com variância complexa ${ }^{13}$.

A análise dos resíduos foi realizada nos dois níveis.
Os resíduos do nível 2 são chamados resíduos reduzidos e são obtidos multiplicando-se os resíduos brutos, oriundos da regressão multinível, pelo fator de redução (FR):

$$
\mathrm{FR}=\frac{\sigma_{\mathrm{u} 0}^{2}}{\sigma_{\mathrm{u} 0}^{2}+\sigma_{\mathrm{e} 0}^{2} / \mathrm{N}_{\mathrm{n}}}
$$

em que $\mathrm{N}_{\mathrm{n}}$ é o número de unidades do nível 1 no grupo de nível 2, e o FR pode assumir valores entre 0 e 1 14,15.

Foi avaliado o gráfico dos resíduos reduzidos ordenados com intervalo de 95\% de confiança. Os resíduos representam os afastamentos das estimativas médias das microrregiões em relação à média geral predita para o intercepto e para a inclinação 15 .

As análises multiníveis e de dados de painel foram realizadas com o auxílio dos aplicativos computacionais MLwiN 2.02 (Centre for Multilevel Modelling, Bristol, Reino Unido) e SAS 9.1 (SAS Inst., Cary, Estados Unidos), respectivamente.

\section{Questões éticas}

Este estudo foi aprovado pela Comissão de Pesquisa da Faculdade de Medicina da Universidade Federal do Rio Grande do Sul, atendendo às exigências recomendadas pela Resolução no. 196/96 do Conselho Nacional de Saúde.

\section{Resultados}

A taxa de crescimento anual das proporções de baixo peso ao nascer para o Rio Grande do Sul é $1,2 \%$, obtida da modelagem exponencial.

O menor valor para a proporção de baixo peso ao nascer, no período de 1994-2004, foi de 4,2\% na microrregião de Cerro Largo, no ano de 1997. No outro extremo, a proporção de baixo peso ao nascer de $11,5 \%$ na microrregião de Carazinho, em 2004.

As médias das proporções de baixo peso ao nascer para as 35 microrregiões são apresentadas na Figura 1. Destaca-se a microrregião de Carazinho, ao norte do estado, como a de maior média da proporção de baixo peso ao nascer $(10,1 \%)$, e as microrregiões de Santa Rosa, Cerro Largo, Sananduva, também no norte, e Montenegro, mais a leste, como as de menores médias.

Avaliando a evolução das proporções de baixo peso ao nascer nos 11 anos para todas as microrregiões, pode-se observar uma tendência de aumento, de uma maneira geral, embora haja variabilidade de comportamento entre as microrregiões. Observa-se que $74 \%$ das microrregiões têm o valor da proporção de baixo peso 

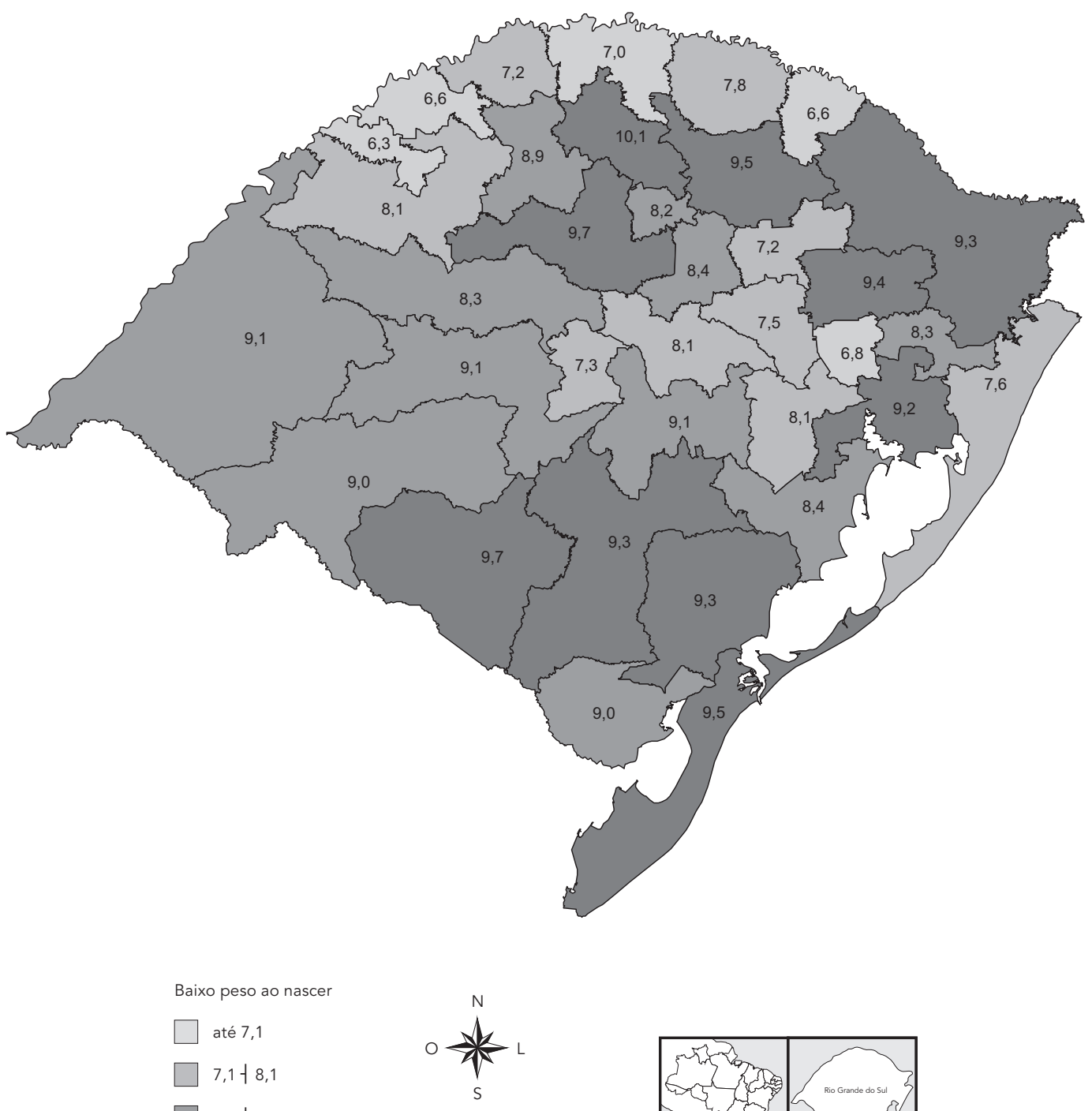

$8,1+9,1$

$9,1+10,1$

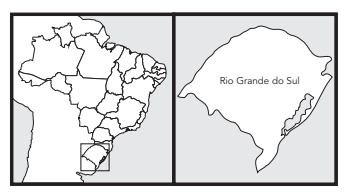

ao nascer do ano de 2004 superior à média dos 11 anos.

Na Tabela 1, são apresentados os valores médios e extremos das variáveis que permaneceram no modelo multinível final. É interessante observar a variabilidade desses indicadores nas microrregiões nesse período, justificando seu poder preditivo nos modelos ajustados.
Na Tabela 2 estão os valores para a proporção de baixo peso ao nascer e variáveis do nível individual, para os anos 1994 e 2004 no Rio Grande do Sul.

Na Tabela 3, são apresentados a análise via modelos mistos (Modelo 1) e os quatro modelos multiníveis estimados. O Modelo 1 é o modelo de dados de painel com estrutura de covariância 
Estatísticas descritivas das variáveis incluídas no modelo multinível, segundo os níveis individual e de contexto.

\begin{tabular}{|c|c|c|c|}
\hline Variáveis & Média \pm DP & Mínimo & Máximo \\
\hline \multicolumn{4}{|l|}{ Nível 1 (anos) } \\
\hline Baixo peso ao nascer (\%) & $8,37 \pm 1,27$ & 4,22 & 11,45 \\
\hline Pré-termo (\%) & $6,16 \pm 2,41$ & 1,79 & 19,34 \\
\hline $\begin{array}{l}\text { Coeficiente de mortalidade infantil } \\
\text { (/1.000 nascidos vivos) }\end{array}$ & $16,84 \pm 4,75$ & 3,55 & 33,80 \\
\hline Cesáreas (\%) & $43,84 \pm 6,90$ & 27,90 & 62,22 \\
\hline \multicolumn{4}{|l|}{ Nível 2 (microrregiões) } \\
\hline Urbanização 2000 (\%) & $70,34 \pm 14,19$ & 42,79 & 96,51 \\
\hline Participação 2000 (\%) & $61,59 \pm 5,37$ & 52,28 & 69,64 \\
\hline Gasto SUS 2003 (R\$) & $61,74 \pm 25,64$ & 35,37 & 165,27 \\
\hline
\end{tabular}

DP: desvio-padrão.

Valores e variação do baixo peso ao nascer e de seus indicadores no nível individual, para os anos de 1994 e 2004 , Rio Grande do Sul, Brasil.

\begin{tabular}{lccc}
\hline Variáveis & $\mathbf{1 9 9 4}$ & $\mathbf{2 0 0 4}$ & Variação no período \\
\hline Baixo peso ao nascer (\%) & 8,6 & 9,3 & $+0,7$ \\
Pré-termo (\%) & 5,4 & 8,5 & $+3,1$ \\
Coeficiente de mortalidade infantil & 19,2 & 15,2 & $-4,0$ \\
(/1.000 nascidos vivos) & & 47,2 & $+9,2$ \\
Cesáreas (\%) & 38,0 & 4 & \\
\hline
\end{tabular}

auto-regressiva de $1 \underline{\text { a }}$ ordem e médias móveis (ARMA $(1,1))$, sendo aquele que apresentou a menor deviance $(147,22 ; \mathrm{p}<0,0001)$, em que a variável cesárea, do nível 1, não é significativa. Os modelos multiníveis 2, 3 e 4 são de componentes de variância, e o modelo 5 é de coeficientes aleatórios, com variância complexa.

A estimativa da média geral para as proporções de baixo peso ao nascer foi obtida do Modelo 2 (modelo nulo), sendo 8,37\% (intercepto) e tendo uma correlação intragrupo de $62,2 \%$, ou seja, $62,2 \%$ da variação das proporções de baixo peso ao nascer se devem às microrregiões, conseqüentemente, $37,8 \%$ é em razão dos anos.

A inclusão das variáveis significativas de nível 1 resultou no Modelo 3, com o intercepto aleatório, ocorrendo uma redução de $21,7 \%$ na variância do nível 2 e de 27,4\% na variância do nível 1 . Avaliando o coeficiente estimado para a variável anos (centrada no ano de 1994), constata-se que as proporções de baixo peso ao nascer aumentam com o tempo.

No Modelo 4, com intercepto aleatório e com todas as variáveis significativas do nível 1 e do nível 2, pode-se observar uma grande redução na variância do nível $2(73,7 \%)$ e moderada redução no nível 1 (27,6\%). Nesse modelo, verifica-se que as variáveis preditoras explicaram a maior parte da variação das proporções de baixo peso ao nascer em razão do nível das microrregiões.

Os Modelos 4 e 5 informam que a proporção de baixo peso ao nascer aumenta com os anos, de 1994-2004, nas microrregiões. Isso pode ser explicado pelo aumento do percentual de recémnascidos pré-termo, do percentual de partos cesáreos, do coeficiente de mortalidade infantil, da taxa de urbanização, dos gastos com o SUS e pelo decréscimo do percentual da participação (população economicamente ativa) nas microrregiões.

No Modelo 5, os interceptos (médias das proporções de baixo peso ao nascer entre as microrregiões) variam entre as microrregiões, e as inclinações variam entre as microrregiões em função dos anos.

Estima-se que, em média, o aumento de $1 \%$ no percentual de prematuros por ano acarreta o acréscimo de $0,057 \%$ na proporção de baixo peso 
Modelos de painel e multiníveis para a proporção de baixo peso ao nascer, com efeitos das co-variáveis no nível dos anos (1994-2004) e das microrregiões do Rio Grande do Sul, Brasil.

\begin{tabular}{|c|c|c|c|c|c|}
\hline \multirow[t]{2}{*}{ Variáveis } & \multirow{2}{*}{$\begin{array}{c}\text { Análise de dados } \\
\text { de painel } \\
\text { Modelo } 1\end{array}$} & \multicolumn{4}{|c|}{ Análise de regressão multinível } \\
\hline & & Modelo 2 & Modelo 3 & Modelo 4 & Modelo 5 \\
\hline Efeitos fixos & & \multicolumn{4}{|c|}{ Coeficiente (erro padrão) * } \\
\hline Intercepto & $9,476(1,806)$ & $8,370(0,174)$ & $7,992(0,170)$ & $8,012(0,117)$ & $7,991(0,113)$ \\
\hline \multicolumn{6}{|l|}{ Nível 1} \\
\hline Anos (centrados em 1994) & $0,084(0,017)$ & & $0,076(0,014)$ & $0,072(0,014)$ & $0,077(0,017)$ \\
\hline Pré-termo (\% prematuros) & $0,073(0,021)$ & & $0,075(0,021)$ & $0,071(0,020)$ & $0,057(0,021) p=0,007$ \\
\hline $\begin{array}{l}\text { Coeficiente de mortalidade infantil } \\
\text { (mortes/1.000 nascidos vivos) }\end{array}$ & $0,058(0,010)$ & & $0,064(0,010)$ & $0,060(0,010)$ & $0,060(0,011)$ \\
\hline \multirow[t]{2}{*}{ Cesáreas (\%) } & - & & $0,018(0,011)$ & $0,026(0,010)$ & $0,020(0,010)$ \\
\hline & & & & $p=0,011$ & $p=0,046$ \\
\hline \multicolumn{6}{|l|}{ Nível 2} \\
\hline \multirow[t]{2}{*}{ Urbanização 2000 (\% população) } & $0,025(0,009)$ & & - & $0,023(0,008)$ & $0,022(0,009)$ \\
\hline & $p=0,013$ & & & $p=0,006$ & $p=0,008$ \\
\hline Participação 2000 (\% população & $-0,070(0,023)$ & & - & $-0,080(0,022)$ & $-0,080(0,022)$ \\
\hline economicamente ativa) & $p=0,005$ & & & & \\
\hline Gastos SUS 2003 (R\$ investimento & $0,010(0,005)$ & & - & $0,010(0,004)$ & $0,010(0,004)$ \\
\hline em saúde) & $p=0,028$ & & & $p=0,011$ & $p=0,019$ \\
\hline \multicolumn{6}{|l|}{ Efeitos aleatórios } \\
\hline Variância dos interceptos $\left(\sigma^{2} \mathrm{u0}\right)$ & - & $1,007(0,254)$ & $0,788(0,198)$ & $0,265(0,073)$ & $0,240(0,076)$ \\
\hline \multirow[t]{2}{*}{ Variância das inclinações $\left(\sigma_{\mathrm{u} 1}^{2}\right) * \star$} & - & & - & - & $0,003(0,001)$ \\
\hline & & & & & $p=0,033$ \\
\hline Variância do nível $1\left(\sigma^{2} \mathrm{e}\right)$ & - & $0,613(0,046)$ & $0,445(0,034)$ & $0,444(0,034)$ & $0,325(0,036)$ \\
\hline \multirow[t]{2}{*}{ Variância do nível $1\left(\sigma^{2}{ }_{\mathrm{e}}\right)^{* \star}$} & - & & - & - & $0,004(0,002)$ \\
\hline & & & & & $p=0,022$ \\
\hline -2 log verossimilhança & 884,650 & $1.007,171$ & 886,218 & 851,131 & 829,766 \\
\hline
\end{tabular}

* Coeficientes sem o valor da significância correspondem a $p<0,001$;

** Variância complexa (nível 2 função dos anos; nível 1 função do coeficiente de mortalidade infantil).

Modelo 1: de painel via modelos mistos; modelo 2: nulo, sem variáveis; modelo 3: componente de variância, com variáveis do nível 1; modelo 4: componente de variância, com variáveis do nível 1 e 2; modelo 5: coeficientes aleatórios, com variáveis nos dois níveis e variância complexa.

ao nascer; o aumento de $1 \%$ o no coeficiente de mortalidade infantil implica acréscimo de $0,06 \%$ na proporção de baixo peso ao nascer, e o aumento de $1 \%$ no percentual de cesáreas implica acréscimo de $0,02 \%$ na proporção de baixo peso ao nascer.

No nível das microrregiões, pode-se dizer que, a cada aumento de $1 \%$ na urbanização das microrregiões, ocorre o aumento de $0,022 \%$ na proporção de baixo peso ao nascer; o aumento de $1 \%$ no percentual de pessoas economicamente ativas em idade produtiva implica um decréscimo de $0,08 \%$ na proporção de baixo peso ao nascer, e o aumento de $\mathrm{R} \$ 1,00$ nos gastos com o SUS provoca um aumento de $0,01 \%$ na proporção de baixo peso ao nascer.

A inclusão de variância complexa no modelo indica que a variância das proporções de baixo peso ao nascer entre as microrregiões (nível 2) foi modelada em função dos anos, aumentando com eles, e, para o nível 1, a variância das proporções de baixo peso ao nascer é em função do coeficiente de mortalidade infantil e varia com ele. Para microrregiões com menores coeficientes de mortalidade infantil, ou seja, regiões com melhores condições de saúde, a variância da proporção de baixo peso ao nascer nos anos diminui com o aumento do coeficiente de mortalidade infantil, e, para microrregiões com maiores coeficientes de mortalidade infantil, regiões com piores condições de saúde, a variância da proporção de baixo peso ao nascer aumenta com o aumento do coeficiente de mortalidade.

Tanto no nível 2 como no nível 1, os termos de covariância não foram significativos, sendo retirados do modelo. Nesse caso, não existe cor- 
relação significativa entre os termos de erro nos níveis.

As variâncias do Modelo 5, embora tenham reduzido consideravelmente em relação ao modelo nulo, ainda são estatisticamente significativas.

Avaliando os resíduos reduzidos ordenados do nível 2 para o intercepto, observa-se que $4 \mathrm{mi}$ crorregiões (Montenegro, Sananduva, Santa Rosa, Cerro Largo) apresentam resíduos abaixo da média geral estimada $(7,99 \%)$ para a proporção de baixo peso ao nascer global, indicando que essas microrregiões apresentam médias estimadas de proporção de baixo peso ao nascer para o período de 11 anos, significativamente menores do que 7,99\%, e 3 microrregiões (Ijuí, Vacaria, Carazinho) apresentam resíduos estimados significativamente maiores, isto é, médias de proporção de baixo peso ao nascer maiores do que $7,99 \%$.

Considerando os resíduos reduzidos ordenados do nível 2 para a inclinação, encontra-se que a microrregião de Santa Rosa apresenta resíduo menor que a inclinação média geral estimada $(0,08 \%)$, ou seja, para essa microrregião, a proporção de baixo peso ao nascer aumenta menos com os anos do que a média geral. Já a microrregião de Cachoeira do Sul tem resíduo maior que a média geral estimada, vale dizer, as proporções de baixo peso ao nascer têm um aumento maior com os anos do que o aumento médio.

\section{Discussão}

Há grande variação das proporções de baixo peso ao nascer entre as microrregiões do Rio Grande do Sul. É interessante notar que isso ocorre entre microrregiões que se situam na mesma mesorregião, como é o caso da Noroeste Rio-Grandense.

De uma maneira geral, há uma tendência de aumento das proporções de baixo peso ao nascer, comprovada pela proporção estimada para o Rio Grande do Sul e pela constatação de que 26 das 35 microrregiões (74\%) têm o valor da proporção de baixo peso ao nascer do ano de 2004 superior à média dos 11 anos avaliados. De acordo com tais resultados, é pequena a chance de que o Rio Grande do Sul consiga reduzir a incidência de baixo peso ao nascer contribuindo para a redução da mortalidade infantil, que é um dos Objetivos de Desenvolvimento do Milênio 4.

Os resultados obtidos pela modelagem de dados de painel via modelos mistos identificaram a maioria dos preditores encontrados com o modelo multinível, com exceção do percentual de cesarianas.

A inclusão do percentual de cesarianas no modelo multinível e a não-inclusão no modelo de dados de painel se deve, em parte, à significância da variável, que foi próxima a 0,05 , e à estrutura do modelo multinível.

A análise de medidas repetidas, utilizando os modelos multiníveis, considerou as medidas nos anos (nível 1) pertencendo a uma mesma microrregião (nível 2), com as microrregiões constituindo um dos níveis de hierarquia 9 . Assim, o modelo multinível acomodou a hierarquia dos dados na estrutura do modelo e estimou com mais eficiência as variâncias dos coeficientes, bem como os fatores de risco do nível da microrregião 10 . A maior diferença encontrada na estimação dos coeficientes fixos dos modelos de dados de painel e multinível foi no intercepto do modelo.

Neste estudo, foi modelada a estrutura de variância dos dois níveis da hierarquia, ou seja, em vez de tentar estabilizar a variância utilizando transformações, ela foi modelada em função das variáveis preditoras do modelo 9 .

O modelo multinível adotado neste estudo permitiu concluir que: (1) há maior variação nas proporções de baixo peso ao nascer entre as microrregiões do que entre os anos; (2) os coeficientes fixos do nível individual explicam parte da variação das proporções de baixo peso ao nascer entre os anos, e as proporções de baixo peso ao nascer estimadas aumentam com o aumento dos valores dos preditores do nível individual; (3) o coeficiente de mortalidade infantil é um preditor significativo para as proporções de baixo peso ao nascer, mostrando que a associação entre as proporções de baixo peso ao nascer e os coeficientes de mortalidade infantil ocorre nas duas direções, pois o baixo peso ao nascer é um conhecido preditor da mortalidade infantil, e o coeficiente de mortalidade infantil é um indicador de saúde da população. Segundo Duarte 7, o coeficiente de mortalidade infantil reflete o nível de saúde, condições de vida e desenvolvimento sócio-econômico de uma população, isto é, quanto maior o coeficiente de mortalidade infantil de uma localidade maior a proporção de baixo peso ao nascer; (4) os resíduos reduzidos estimados representam a parcela da variância contextual que este modelo não conseguiu explicar. Os resíduos possibilitam identificar que Montenegro, Sananduva, Santa Rosa e Cerro Largo são microrregiões com situação mais positiva em relação aos recém-nascidos. Já as microrregiões de Ijuí, Vacaria e Carazinho apresentam situação pior do que a média, apontando para a necessidade urgente de intervenção no que diz respeito à saúde dos recém-nascidos dessas localidades; (5) este modelo conseguiu explicar parte da discrepância entre as proporções de baixo peso ao nascer que ocorrem no Rio Grande do Sul. As demais microrregiões não apresentaram resíduos significativos, 
indicando que, para elas, os preditores do modelo explicam satisfatoriamente a variabilidade entre as proporções de baixo peso ao nascer.

A vantagem dos modelos multiníveis ou de coeficientes aleatórios é identificar que a média das proporções de baixo peso ao nascer para cada microrregião difere, e que a relação entre as características individuais e as proporções de baixo peso ao nascer também diferem entre as microrregiões, pois para algumas microrregiões o aumento nas proporções de baixo peso ao nascer com os anos é mais pronunciado do que em outras. Levar em conta tais diferenças nas inclinações permite diferenciar as microrregiões.

Antes do surgimento das análises multiníveis, havia duas opções no tratamento dos dados: as características do nível individual poderiam ser agregadas no nível mais alto de hierarquia, ou as características de níveis mais altos eram distribuídas para todas as unidades do nível individual. Agregar ou distribuir essas características causa problemas na interpretação dos resultados, conhecidos como falácia atomística e falácia ecológica, respectivamente. A falácia atomística é a falácia de fazer inferências no nível de grupo baseado em dados do nível individual. A falácia ecológica é a falácia de fazer inferências no nível individual baseado em dados do nível de grupo 5,16 .

A utilização dos modelos multiníveis permite testar suposições sobre a relação entre características no nível de contexto e o desfecho, levando em conta as influências individuais 16 .

Em termos práticos, a conseqüência de ignorar a estrutura multinível dos dados é encontrar muitas relações significativas erroneamente 16 .

A maior contribuição deste estudo em relação aos que utilizam as análises tradicionais é que o modelo multinível levou em conta a estrutura de hierarquia dos dados na modelagem, influenciando na estimação dos coeficientes do modelo.

Alguns artigos relevantes encontrados na literatura sobre proporções de baixo peso ao nascer podem servir de base para comparações, mas nenhum deles com modelagem multinível.

O trabalho de Victora et al. ${ }^{2}$ sobre proporções de baixo peso ao nascer no Rio Grande do Sul no período de 1980 a 1992 considerou o estado dividido em Delegacias Regionais de Saúde. Os autores concluíram, para aquele período, que a estabilidade nas proporções de baixo peso ao nascer sugeria que as condições gerais das gestantes, relativas ao estado nutricional e infecções, assim como do atendimento pré-natal, não tinham apresentado progresso naquela década.

A análise geográfica dos indicadores de baixo peso ao nascer mostrou uma distribuição desi- gual dentro do estado, com as regiões Sul, Oeste e Metropolitana apresentando as condições de saúde mais precárias. Já a Região Norte mostrou as melhores condições de saúde 2,17. Essas desigualdades regionais são novamente comprovadas no presente estudo.

Monteiro et al. 18, estudando a evolução do peso ao nascer na cidade de São Paulo (1976-1998), encontraram evolução favorável nos estratos de baixo nível socioeconômico, aparentemente, por conta do melhor desempenho do crescimento intrauterino, o qual poderia decorrer de melhorias nas condições socioeconômicas, no peso e na altura das gestantes, na assistência pré-natal e, possivelmente, no declínio do hábito de fumar. Nos estratos de alto nível socioeconômico ocorreu uma evolução desfavorável, aparentemente, por causa do aumento da proporção de prematuros 18, tendência provocada pelo aumento da proporção de cesarianas.

Um estudo sobre nascidos vivos em unidade hospitalar do Recife, em Pernambuco (19912000), concluiu pela tendência progressiva das taxas de cesarianas, de baixo peso ao nascer, de prematuridade e de mães adolescentes. Nesse estudo, os autores encontraram a maior proporções de baixo peso ao nascer $(22,8 \%)$ para o ano de 2000, e uma elevada taxa de cesáreas, variando de 29,4\% em 1993 a 35,2\% em 2000 19. Tais resultados corroboram com os achados no presente estudo, em que o aumento das taxas de cesáreas e da prematuridade se associam ao aumento das proporções de baixo peso ao nascer.

Barros et al. 20 referem que o aumento nas proporções de baixo peso ao nascer em Pelotas, em 1993, foi decorrente tanto da maior proporção de nascidos pré-termo quanto de crianças com restrição de crescimento intrauterino (nascidos vivos a termo).

Em outro artigo publicado por Barros et al. 21 com dados de três coortes de crianças nascidas na cidade de Pelotas, no Rio Grande do Sul, os autores encontraram proporções de baixo peso ao nascer de $9 \%, 9,8 \%$ e $10,4 \%$ para os anos de 1982, 1993 e 2004, respectivamente, estando em concordância com o presente estudo, ou seja, as proporções de baixo peso ao nascer vêm aumentando com os anos, o que também foi constatado em outros artigos 2,22,23.

A cidade de Pelotas localiza-se na mesorregião sudeste Rio-Grandense, onde as proporções de baixo peso ao nascer das microrregiões são mais elevadas. Considerando que a expansão da assistência à saúde em Pelotas levou mais de 98\% de mulheres grávidas a receberem cuidados prénatais, com uma média de 8,3 consultas por mulher em 2004, é paradoxal que as proporções de baixo peso ao nascer tenham aumentado. Uma 
possível explicação seria a melhoria na qualidade do diagnóstico e do atendimento às gestantes, possibilitando a maior intervenção na gestação (cesariana e indução de parto), quando necessário.

Mariotoni et al. 24 estudaram o peso ao nascer na Maternidade de Campinas ao longo de 25 anos, não encontrando variação das proporções de baixo peso ao nascer, que ficaram em torno de $8 \%$. O estudo concluiu que, embora as condições gerais da população tenham melhorado - condição importante para a melhoria da saúde - não foi encontrada evolução positiva no peso ao nascer, provavelmente por outras modificações que impediram esse avanço.

Para o Estado de Sergipe, os valores das proporções de baixo peso ao nascer foram 10,8\% e $7 \%$ para 1988 e 1998, respectivamente, mostrando um comportamento decrescente no período, sendo provocado possivelmente pela melhoria na qualidade de vida 25 . Esses resultados diferem de outros trabalhos encontrados na literatura para outros estados brasileiros. Uma explicação possível para o aumento das proporções de baixo peso ao nascer é que a interrupção da gestação pela indução ou parto cesáreo tem-se tornado mais frequente 17,21 . O uso de novas técnicas para prevenir a mortalidade perinatal e neonatal em países desenvolvidos tem sido, em parte, responsável pelo aumento observado de recémnascidos pré-termo 21 . Também, o aumento do uso do ultrassom pode ter contribuído para o aumento do número de partos prematuros 20,26, o que indica melhoria nos diagnósticos ou erro na avaliação da idade gestacional por esse exame. No Rio Grande do Sul (também nas regiões Sul e Sudeste) os bebês prematuros constituem mais da metade da população de baixo peso ao nascer, e este percentual vem aumentando ano a ano. Em 2000, era de 53\% e atingiu 58\% em 2007 (Informações em saúde: estatísticas vitais. http:// www.datasus.gov.br, acessado em 29/Jul/2006).

Freitas et al. 17 concluem que as altas taxas de cesariana no sul do Brasil constituem problema de saúde pública e estão associadas a fatores sociais, econômicos e culturais, os quais podem levar ao mau uso da tecnologia médica na atenção ao parto.

O estudo de Andrade et al. 27 corrobora o achado deste estudo de associação entre urbanização e baixo peso ao nascer. Os autores encontraram maior proporção de baixo peso ao nascer nas regiões mais desenvolvidas, confirmando o paradoxo do baixo peso ao nascer.

Um estudo ecológico, com dados dos estados americanos (Estados Unidos) nos anos de 19851995, encontrou um aumento uniforme nas proporções de baixo peso ao nascer, que esteve associado negativamente à urbanização e aos cuidados pré-natais, entre outros preditores 28 , discordando do efeito positivo da urbanização sobre as proporções de baixo peso ao nascer e do número de consultas pré-natais, que não esteve associado às proporções de baixo peso ao nascer no presente estudo. Os autores concluíram que os cuidados pré-natais contínuos e abrangentes e cuidados básicos de saúde e de orientações na vida da mulher podem promover melhores desfechos na saúde. Também concluíram que residir em áreas metropolitanas possibilita maiores recursos na atenção básica à saúde, ainda que nessas áreas exista grande desigualdade social no acesso aos recursos, sobretudo para mulheres afro-americanas 28 . A divergência possivelmente está associada à maior abrangência e melhor qualidade da assistência pré-natal nos Estados Unidos em relação ao Brasil.

Outro estudo sobre a evolução do peso ao nascer, na Espanha, encontrou um aumento linear das proporções de baixo peso ao nascer entre os anos de 1981 e 2002. Os autores avaliaram que alguns fatores sociais como o aumento do número de gestantes fumantes, entre outros, poderiam estar afetando o peso ao nascer 29.

As limitações encontradas neste estudo foram: a utilização de dados secundários; o grande tamanho das microrregiões, apresentando alta variabilidade interna; e os indicadores específicos para as microrregiões disponíveis, restringindo as informações para a modelagem.

Portanto, este estudo aponta na direção do uso da modelagem multinível em áreas geográficas menores, possivelmente apresentando menor variação interna no nível 2 , e também na busca de indicadores mais específicos para explicar a alta incidência do baixo peso ao nascer no Rio Grande do Sul.

Indica que efeitos contextuais, como a urbanização e os investimentos na saúde, têm efeitos negativos no peso ao nascer, ou seja, maiores investimentos em saúde que resultam em diagnósticos mais precoces e de melhor qualidade, intervenção na gravidez e no parto, com o objetivo de salvar o feto, levam ao aumento das proporções de baixo peso ao nascer. É possível que o aumento das proporções de baixo peso ao nascer tenha direcionado para o aumento nos investimentos em saúde na tentativa de diminuí-las, indicando um caminho inverso ao encontrado pelo modelo. Também deve ser considerado que os investimentos em atenção básica não necessariamente significam um atendimento pré-natal abrangente e de qualidade que poderia levar à redução dos resultados adversos da gestação 27 .

O estudo orienta, também, para as condições socioeconômicas da região - e nele está incluída 
a participação ativa na vida econômica (escolaridade, empregos etc.) - que produzem uma redução nas proporções de baixo peso ao nascer.

Em relação aos aspectos específicos com o cuidado à saúde, deve-se levar em conta o percentual de cesarianas, de partos prematuros e o coeficiente de mortalidade infantil, que reflete a realidade da saúde da região.

O Rio Grande do Sul, considerado o estado com a melhor qualidade de vida dentre os estados brasileiros, necessita investir esforços na ampliação dos cuidados integrais à mulher, iniciando pela educação e cuidados básicos na sua saúde desde a infância, e incluindo a melhora na qualidade e quantidade dos cuidados pré-natais. A melhoria na qualidade da atenção pré-natal está relacionada às orientações quanto ao uso do fumo, drogas, álcool, alimentação etc., bem como ao acompanhamento da gestante desde o início da gestação até a atenção ao parto.

Os fatores que influenciam a saúde não são modificados rapidamente, mas podem ser trabalhados para resultados melhores em médio e em longo prazo. Tanto os programas de saúde quanto a população devem trabalhar a fim de proteger a mulher e seu filho, podendo assim interferir nos fatores que comprometem o peso do recém-nascido 24 .

\section{Resumo}

O objetivo deste estudo ecológico longitudinal foi analisar a tendência da proporção de baixo peso ao nascer no Rio Grande do Sul, Brasil, de 1994 a 2004, utilizando a análise de dados de painel e regressão linear multinível (dois níveis: microrregião e tempo (anos)) para estimar os fatores de risco associados à proporção de baixo peso ao nascer. A proporção de baixo peso ao nascer teve um crescimento anual de 1,2\%, e o modelo multinível mostrou que as proporções diferem entre as microrregiões e aumentam em associação com os anos, com o aumento do percentual de prematuros, com o aumento do coeficiente de mortalidade infantil e com o aumento do percentual de cesarianas. Entre as microrregiões, as proporções de baixo peso ao nascer variam positivamente com o percentual de urbanização, com os gastos com o Sistema Único de Saúde e negativamente com o percentual de participação na atividade econômica. O modelo multinível mostrou que a maior parte da variação nas proporções de baixo peso ao nascer se deve aos efeitos da microrregião de moradia da mãe do nascido vivo.

Sistemas de Informação; Recém-Nascido de Baixo Peso; Análise Multinível

\section{Colaboradores}

A. B. Moraes e R. R. Zanini contribuíram com o delineamento, coleta dos dados, análise estatística, interpretação e redação do artigo. E. R. J. Giugliani e J. Riboldi colaboraram na orientação, redação e revisão do artigo. 


\section{Referências}

1. Kramer MS. Determinants of low birth weight: methodological assessment and meta-analysis. Bull World Health Organ 1987; 65:663-737.

2. Victora CG, Grassi PR, Schmidt AM. Situação de saúde da criança em área da região Sul do Brasil, 1980-1992: tendências temporais e distribuição espacial. Rev Saúde Pública 1994; 28:23-32.

3. World Health Organization. Towards the development of a strategy for promoting optimal fetal growth. Geneva: World Health Organization; 2004.

4. Mendonça R. Em direção às metas de desenvolvimento do milênio: uma análise regional. http:// www.uff.br/econ/download/tds/UFF_TD179.pdf (acessado em 21/Nov/2005).

5. Diez-Roux AV. Bringing context back into epidemiology: variables and fallacies in multilevel analysis. Am J Public Health 1998; 88:216-22.

6. Susser M. The logic in ecological: I. the logic of analysis. Am J Public Health 1994; 84:825-9.

7. Duarte CMR. Reflexos das políticas de saúde sobre as tendências da mortalidade infantil no Brasil: revisão da literatura sobre a última década. Cad Saúde Pública 2007; 23:1511-28.

8. Bryk AS, Raudenbush SW. Hierarchical linear models. Newbury Park: Sage Publications; 1992.

9. Brown H, Prescott R. Applied mixed models in medicine. New York: John Wiley \& Sons, 2001.

10. Snijders T, Bosker R. Multilevel analysis: an introduction to basic and advanced multilevel modeling. London: Sage Publications; 1999.

11. Goldstein H. Multilevel statistical models. London: Arnold; 2003.

12. Leyland AH, McLeod A. Mortality in England and Wales, 1979-1992. An introduction to multilevel modeling using MLwiN. http://www.soziologie. uni-halle.de/langer/multilevel/progs/mlwin/ tutoriall.pdf (acessado em 01/Abr/2006).

13. Barros AJD. Modelos multinível: primeiros passos. Pelotas: Departamento de Medicina Social, Faculdade de Medicina, Universidade Federal de Pelotas; 2001.

14. Merlo J, Chaix B, Yang M, Lynch J, Rästam L. A brief conceptual tutorial of multilevel analysis in social epidemiology: linking the statistical concept of clustering to the idea of contextual phenomenon. J Epidemiol Community Health 2005; 59:443-9.

15. Rasbash J, Steele F, Browne W, Prosser B. A user's guide to MLwiN. Bristol: Centre for Multilevel Modelling, University of Bristol; 2005.

16. Leyland AH, Groenewegen PP. Multilevel modelling and public health policy. Scand J Public Health 2003; 31:267-74.

17. Freitas PF, Drachler ML, Leite JCC, Grassi PR. Desigualdade social nas taxas de cesariana em primíparas no Rio Grande do Sul. Rev Saúde Pública 2005; 39:761-7.
18. Monteiro CA, Benicio MHDA, Ortiz LP. Tendência secular do peso ao nascer na cidade de São Paulo (1976-1998). Rev Saúde Pública 2000; 34(6 Suppl):26-40.

19. Vidal SA, Arruda BKG, Vanderlei LC, Frias PG. Avaliação da série histórica dos nascidos vivos em unidade terciária de Pernambuco - 1991 a 2000. Rev Assoc Med Bras 2005; 51:17-22.

20. Barros FC, Victora CG, Tomasi E, Horta B, Menezes AM, César JA, et al. Saúde materno-infantil em Pelotas, Rio Grande do Sul, Brasil: principais conclusões da comparação dos estudos das coortes de 1982 e 1993. Cad Saúde Pública 1996; 12 Suppl 1:87-92.

21. Barros FC, Victora CG, Barros AJD, Santos IS, Albernaz E, Matijasevich A, et al. The challenge of reducing neonatal mortality in middle-income countries: findings from three Brazilian birth cohorts in 1982, 1993 and 2004. Lancet 2005; 365: 847-54.

22. Horta BL, Barros FC, Halpern R, Victora CG. Baixo peso ao nascer em duas coortes de base populacional no sul do Brasil. Cad Saúde Pública 1996; 12 Suppl 1:27-31.

23. Bettiol H, Rona RJ, Chinn S, Goldani M, Barbieri MA. Factors associated with preterm birth in Southeast Brazil: a comparison of two birth cohort born 15 years apart. Paediat Perinat Epidemiol 2000; $14: 30-8$.

24. Mariotoni GGB, Barros Filho AA. Peso ao nascer e características maternas ao longo de 25 anos na Maternidade de Campinas. J Pediatr (Rio J.) 2000; 76:55-64.

25. Gurgel RQ, Dias IMO, França VLA, Castañeda DFN. Distribuição espacial do baixo peso ao nascer em Sergipe, Brasil, 1995/1998. Cad Saúde Pública 2005; 21:1329-37.

26. Yang H, Kramer MS, Platt RW, Blondel B, Bréart G, Morin I, et al. How does early ultrasound scan estimation of gestational age lead to higher rates of preterm birth? Am J Obstet Gynecol 2002; 186: 433-37.

27. Andrade CLT, Szwarcwald CL, Castilho EA. Baixo peso ao nascer no Brasil de acordo com as informações sobre nascidos vivos do Ministério da Saúde, 2005. Cad Saúde Pública 2008; 24:2564-72.

28. Shi L, Macinko J, Starfield B, Xu J, Regan J, Politzer $\mathrm{R}$, et al. Primary care, infant mortality, and low birth weight in the states of the USA. J Epidemiol Community Health 2004; 58:374-80.

29. Alonso V, Fuster V, Luna F. La evolución del peso al nacer en España (1981-2002) y su relación conlas características de la reproducción. http:/ / www.didac. ehu.es/antropo (acessado em 20/Out/2006).

Recebido em 15/Abr/2010

Versão final reapresentada em 20/Ago/2010

Aprovado em 13/Out/2010 